\title{
REFERENCES
}

1. I. Gelfand, Normierte Ringe, Rec. Math. (Mat. Sbornik) N. S. vol. 9 (1941) pp. 3-24.

2. I. Gelfand and M. Neumark, On the embedding of normed rings into the ring of operators in Hilbert space, Rec. Math. (Mat. Sbornik) N. S. vol. 12 (1943) pp. 197-213.

3. K. G. Wolfson, The algebra of bounded functions, Proc. Amer. Math. Soc. vol. 5 (1954) pp. 10-14.

UNIVERSity OF MichigaN

\section{A NOTE ON UNSTABLE HOMEOMORPHISMS ${ }^{1}$}

ROBERT F. WILLIAMS

In [1] W. R. Utz introduced the concept of an unstable homeomorphism and raised the question of whether there exists an unstable homeomorphism of a compact continuum onto itself. In this note an example of such an homeomorphism will be given.

Let $C$ denote the complex unit circle and for each $z \in C$, let $g(z)=z^{2}$. Then $g: C$ onto $C$ determines an inverse limit space $\Sigma_{2}$ $=\left\{\left(a_{0}, a_{1}, a_{2}, \cdots\right) \mid\right.$ for each non-negative integer $i, a_{i} \in C$ and $\left.g\left(a_{i+1}\right)=a_{i}\right\}$. For $a, b \in \Sigma_{2}$, the function $\rho(a, b)=\sum_{i=0}^{\infty}\left|a_{i}-b_{i}\right| / 2^{i}$ is a metric for $\Sigma_{2} ; \Sigma_{2}$ is familiar as the "two-solenoid," and is a compact, indecomposable continuum. Define $f: \Sigma_{2}$ onto $\Sigma_{2}$ as follows: for each $a=\left(a_{0}, a_{1}, \cdots\right) \in \Sigma_{2}$, let $f(a)=\left[g\left(a_{0}\right), g\left(a_{1}\right), \cdots\right]$. Then $f(a)$ $=\left(a_{0}^{2}, a_{1}^{2}, \cdots\right)=\left(a_{0}^{2}, a_{0}, a_{1}, \cdots\right), f^{-1}(a)=\left(a_{1}, a_{2}, a_{3}, \cdots\right)$, and $f$ is a homeomorphism of $\Sigma_{2}$ onto $\Sigma_{2}$.

To show that $f$ is unstable, suppose that $a=\left(a_{0}, a_{1}, \cdots\right)$ and $b=\left(b_{0}, b_{1}, \cdots\right)$ are distinct points of $\Sigma_{2}$. Consider, as Case 1 , that $a_{0} \neq b_{0}$. Let $e^{i \theta}=a_{0}, e^{i \phi}=b_{0}$, where $0 \leqq \theta, \phi<2 \pi$. Then there exists a non-negative integer $n$ such that the angle between the terminal rays of $2^{n} \theta$ and $2^{n} \phi$ is greater than $\pi / 2$. Then $\rho\left[f^{n}(a), f^{n}(b)\right] \geqq\left|a_{0}^{2^{n}}-b_{0}^{2^{n}}\right|$ $=\left|e^{i 2^{n} \theta}-e^{i 2^{n} \phi}\right|>1$.

Case 2: for some integer $n>0, a_{n} \neq b_{n}$, but $a_{i}=b_{i}$, for $0 \leqq i<n$. Then $f^{-n}(a)=\left(a_{n}, a_{n+1}, a_{n+2}, \cdots\right), f^{-n}(b)=\left(b_{n}, b_{n+1}, b_{n+2}, \cdots\right)$, and there-

Presented to the Society, November 26, 1954; received by the editors June 25, 1954.

1 Research under NSF-G358, National Science Foundation.

${ }^{2}$ A homeomorphism $f$ of a compact metric space $X$ onto $X$ is said to be unstable provided there exists a fixed positive number $\delta$, such that if $x$ and $y$ are distinct points of $X$, then there exists an integer $n$, such that $\rho\left[f^{n}(x), f^{n}(y)\right]$ is greater than $\delta$. 
fore $\rho\left[f^{-n}(a), f^{-n}(b)\right] \geqq\left|a_{n}-b_{n}\right|$, which is equal to 2 , because $a_{n}^{2}=b_{n}^{2}$. Therefore $f$ is unstable.

Other examples. If, instead, we take $C=[0,1]$, and $g: C$ onto $C$ defined by

$$
g(x)=\left\{\begin{array}{llr}
2 x, & \text { for } \quad 0 \leqq x \leqq 1 / 2 \\
2-2 x & \text { for } 1 / 2<x \leqq 1
\end{array}\right.
$$

then the inverse limit space is a well known indecomposable continuum that can be embedded in the plane. The homeomorphism $f$, defined just as above, is unstable relative to the metric defined as above, and thus would be so relative to the metric in the plane. Furthermore, as in both examples, $f$ leaves a point fixed, two such continua could be joined at their fixed point, yielding an example in which the space is a decomposable continuum.

\section{BIBLIOGRAPHY}

1. W. R. Utz, Unstable homeomorphisms, Proc. Amer. Math. Soc. vol. 1 (1950) pp. 769-774.

The University of Virginia 